\title{
Detection of Catechol Using a Biosensor Based on Biosynthesized Silver Nanoparticles and Polyphenol Oxidase Enzymes
}

\author{
S. Sandeep ${ }^{a}$, A.S. Santhosh ${ }^{a}$, N. Kumara Swamy, ${ }^{a, b, *}$ \\ G.S. Suresh ${ }^{c}$ and J.S. Melo ${ }^{d}$ \\ ${ }^{a}$ Department of Chemistry, Sri Jayachamarajendra College of Engineering, \\ Mysuru-570006, India. \\ ${ }^{b}$ JSS Research Foundation, Sri Jayachamarajendra College of Engineering Campus, \\ Mysuru-570006, India \\ ${ }^{c}$ Department of Chemistry and Research Centre, NMKRV College for Women, Jayanagar, \\ Banglore-560011, India \\ ${ }^{d}$ Nuclear Agriculture and Biotechnology Division, Bhabha Atomic Research Centre, \\ Mumbai 400 085, India
}

Received July 17, 2017; accepted January 12, 2018

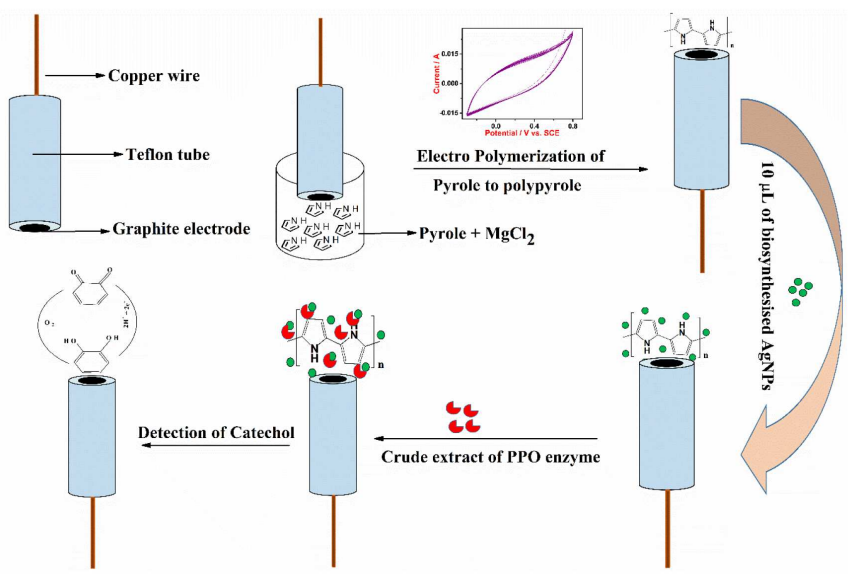

\begin{abstract}
In the present work, we report the development of a polyphenol oxidase (PPO) sensor for the selective and sensitive detection of catechol, using biosynthesized silver nanoparticles (AgNPs). For the sensor development, AgNPs biosynthesized using the leaf extract of Convolvulus pluricaulis, were successfully deposited onto a polypyrrole modified graphite electrode (Gr/PPy). The resulting Gr/PPy/AgNPs electrode was further used as a matrix for the immobilization of the PPO enzyme extracted from Manilkara Zapota (sapota). The morphological characteristics of the developed $\mathrm{Gr} / \mathrm{PPy} / \mathrm{AgNPs} / \mathrm{PPO}$ sensor were studied using a scanning electron microscope (SEM). The sensor performance was evaluated and optimized using cyclic voltammetry (CV), differential pulse voltammetry (DPV), chrono amperometry (CA) and electrochemical impedance spectroscopy (EIS) methods. Under neutral $\mathrm{pH}$ conditions, the developed sensor showed excellent electro catalytic activity towards catechol detection. The sensor
\end{abstract}

\footnotetext{
*Corresponding author. E-mail address: kumaryagati@gmail.com
} 
performed well in the concentration range of 0.001 to $0.015 \mathrm{mM}$, with the detection limit of $0.47 \mu \mathrm{M}$, and sensitivity of $13.66 \mu \mathrm{M}^{-1} \mathrm{~cm}^{-2}$. The biosensor response was found to be uninfluenced by some common interferents, and it also showed good storage stability and repeatability. The practical applicability of the PPO biosensor for catechol detection in real samples was assessed by examining the catechol content in a green tea sample. The sensor could detect catechol content in a green tea sample, to an accuracy of about $98 \%$, thereby establishing its efficiency in real sample analysis.

Keywords: biosynthesized silver nanoparticles, polyphenol oxidase enzyme and catechol.

\section{Introduction}

Catechol (1,2-benzenediol/1,2-dihydroxybenzene) is an important dihydroxybenzene present in many natural sources such as tea, vegetables, fruits and other plants [1-2]. It is considered as one of the key fragments of tea catechins, and its concentration is high up to $18-36 \%$ weight of the fresh dry tea. It is believed to be the basis of many claims made regarding tea's health benefits [3]. Catechol quantification is important, because of its biological roles and environmental significance, in such topics as antioxidation, antivirus, toxicity and carcinogenicity [4-5]. Catechol can be determined using various analytical methods, such as spectrophotometry [6], gas chromatography [7], HPLC [8], etc. However, these techniques have some disadvantages, such as complicated operation, sample pre-treatment, poor selectivity and high cost. On the other hand, sensor technology is one of the most widely adopted in biological and environmental sample analysis, due to its several advantages, such as rapid response, shorter analysis time, no samples pre-treatment, low cost, high sensitivity and selectivity [9]. The sensor performance is, however, dependent of the electrode material and of its composition. PPO has received continuous attention from food chemists and processors, because it is involved in the enzymatic browning of many edible plant products, such as fruits and vegetables [10]. Catechol oxidase or PPO belongs to a group of oxidoreductases, and it is a copper containing an enzyme capable of catalyzing the oxidation of di or polyphenols to the corresponding quinones [11-12]. The positive attributes of high catalytic activity and the ability to utilize different phenolic compounds as substrates have led to a wide interest in its use for the construction of new polyphenol biosensors. The use of plant or fruit extract homogenates, to substitute the isolated enzymes as alternative biologic materials in the biosensors development, has received considerable attention in recent years, as these materials maintain the enzyme of interest in its natural environment, with better enzyme activity and higher stability [13].

The major challenge in the development of enzyme based electrochemical sensors is finding a suitable electrode matrix to immobilize the enzyme without denaturation or modification of its active sites [14].

Metal based nanoparticles such as gold, silver, platinum, copper, palladium, nickel, iron, etc., have attracted considerable attention as electrode materials for the development of ultrasensitive electrochemical sensors, due to their unique 
magnetic, optical and electrocatalytic properties [15]. Biosynthesized metal nanoparticles are interesting materials, as they involve fast, simple and cost effective synthetic processes which do not require high temperatures or toxic chemicals [16]. In particular, biosynthesized AgNPs have gained more attention among the researchers in the field of biosensors, due to their high quantum electron transfer [17] and catalytic properties [18]. AgNPs have high conductivity and a high specific surface area that can adsorb biomolecules such as redox enzyme, proteins and cells, without the loss of activity, thus retaining their functional characteristics to a great extent [19]. Biosynthesized AgNPs can be excellent nontoxic biosensor materials.

Convolvulus pluricaulis is a medicinally important plant, because of its ability to treat a variety of medical conditions, namely, hypertension, neurodegenerative diseases, high blood pressure, epilepsy, vomiting and diabetes [20]. It also enhances memory and decreases cholesterol content in the body. The extract of Convolvulus $p$. plant is also a rich source of chemical constituents, such as alkaloids, phenolic, glycosides, tri-terpenoids and steroids, which reduce the metal salt to form the respective nanoparticles [21]. These reasons have motivated us to use the leaf extract of Convolvulus $p$. plant as a reducing agent for AgNPs biosynthesis.

In the present study, a catechol sensor was developed using a PPO enzyme extracted from Manilkara Zapota and biosynthesized AgNPs, by fabricating them onto a polypyrrole matrix. The developed sensor was successfully used for catechol detection and quantification in a tea sample.

\section{Experimental}

\section{Chemicals and equipments}

Catechol, pyrrole, magnesium chloride and acetonitrile used in the experiments were procured from Sigma Aldrich. For UV-visible spectral studies, Shimadzu $1800 \mathrm{UV}$-visible spectrometer was used. The morphological aspects of the sensor surface were investigated using ZEISS EV040EP (Germany) SEM. CV and DPV were performed using SP-150 Biologic Science Instrument. CA and EIS were performed using Versastat 3 (Princeton Applied Research, USA). The EIS spectral data analysis was carried out using Zswimp win 3.2 simulation software.

\section{AgNPs biosynthesis and characterization}

AgNPs were prepared using Convolvulus $p$. leaves by a procedure earlier reported by our group [22]. $10 \mathrm{~g}$ of freshly collected leaves of this plant were thoroughly washed in deionized water and shade dried. They were crushed in 100 $\mathrm{mL}$ of distilled water, filtered through a sterile muslin cloth, and then through a Whatman-41 filter paper. $10 \mathrm{~mL}$ of this leaf extract were added to $50 \mathrm{~mL}$ of the $0.05 \mathrm{M} \mathrm{AgNO} 3$ aqueous solution, and the mixture was incubated at room temperature, until the solution color changed from pale yellow to dark brown. After the incubation time, the reaction mixture was centrifuged at $10,000 \mathrm{rpm}$ for 15 min, and purified by repeated washing with methanol. Finally, AgNPs were dried at room temperature by evaporating methanol. 


\section{Extraction of the PPO enzyme}

The PPO enzyme used in this study was extracted from Manilkara Z. (sapota) fruit. $25 \mathrm{~g}$ of fruit pulp were homogenized with $25 \mathrm{~mL}$ of $0.05 \mathrm{M}$ phosphate buffer (PBS), with a $\mathrm{pH}$ of $6.8,1 \%$ polyvinyl pyrrolidone, $1 \%$ triton-X 100 and 1 $\mathrm{mM}$ phenyl methyl sulponyl fluoride, for $10 \mathrm{~min}$. The homogenate was rapidly filtered and centrifuged at $10000 \mathrm{rpm}$ for $15 \mathrm{~min}$, at $4{ }^{\circ} \mathrm{C}$. PPO activity was spectrophotometrically determined using 4-methyl catechol as substrate. The enzyme assay was carried out by taking $0.88 \mathrm{~mL}$ of PBS $(\mathrm{pH} 6.8,50 \mathrm{mM})$ and $0.02 \mathrm{~mL}$ of the enzyme extract with $0.1 \mathrm{~mL}$ of catechol as substrate $(0.1 \mathrm{M})$. The increase in absorbance at $420 \mathrm{~nm}$ was monitored for $3 \mathrm{~min}$ using a spectrophotometer, and the average change in absorbance per minute was calculated.

One unit of enzyme activity was defined as the increase in the enzyme amount that caused a change in absorbance of $0.1 \mathrm{~min}^{-1}$ at $420 \mathrm{~nm}$ for the o-quinone produced. The enzyme activity was found to be 620 enzyme units (EU). The extracted enzyme did not show any increase in absorbance when tyrosine was used as substrate. This indicates that there was no monophenolase activity and, therefore, the extracted enzyme is a catecholase. The resulting supernatant was stored at $4{ }^{\circ} \mathrm{C}$ in a refrigerator, and used as a PPO source for further experiments.

\section{Fabrication and characterization of the PPO biosensor}

A graphite electrode was fabricated by inserting a cylindrical graphite rod (Gr) of $6 \mathrm{~mm}$ diameter into a teflon holder with the same internal diameter. A copper wire was used to establish electrical contact. The $\mathrm{Gr}$ electrode surface was polished using a PK-3 electrode polishing kit $(0.05 \mu \mathrm{m}$ aqueous polishing alumina and $1 \mu \mathrm{m}$ polishing diamond), until a mirror shining surface was obtained; finally, it was several times sonicated to remove loosely bound particles, later rinsed with Milli-Q water, and dried with nitrogen gas. The pyrrole monomer was electro-polymerized on the Gr electrode to polypyrrole, by the previously described method [23], with a slight modification. Pyrrole electropolymerization on the $\mathrm{Gr}$ electrode was achieved by immersing the electrode in $20 \mathrm{~mL}$ of acetonitrile containing $0.134 \mathrm{~g}$ of pyrrole and $0.406 \mathrm{~g}$ of $\mathrm{MgCl}_{2}$. Subsequently, the electrode was subjected to cyclic scanning in the range of -0.3 to $0.8 \mathrm{~V}$, at a scan rate of $50 \mathrm{mVs}^{-1}$ for 10 potential cycles, which resulted in PPy deposition on the Gr surface. The graphite surface was then rinsed with acetonitrile to remove an unreacted or loosely bound monomer, and then allowed to dry at room temperature. Further, a suspension of biosynthesized AgNPs in deionized water (w/v ratio of $10 \mathrm{mg}$ of AgNPs in $1 \mathrm{~mL}$ ) was prepared, and $10 \mu \mathrm{L}$ were drop casted over the Gr/PPy electrode surface, and dried at room temperature, to get the Gr/PPy/AgNPs electrode matrix. Finally, PPO was immobilized by drop casting $10 \mu \mathrm{L}$ of the enzyme $\left(620 \mathrm{UmL}^{-1}\right)$ onto the $\mathrm{Gr} / \mathrm{PPy} / \mathrm{AgNPs}$ electrode matrix, to get the modified Gr/PPy/AgNPs/PPO electrode. The fabricated electrode was dried in a refrigerator for $30 \mathrm{~min}$, and later washed in deionized water, to remove loosely bound enzyme particles from 
the electrode surface. The fabricated Gr/PPy/AgNPs/PPO sensor was stored at 4 ${ }^{\circ} \mathrm{C}$, to be used in further studies.

\section{Electrochemical studies}

All electrochemical experiments were performed using a three electrode system with modified $\mathrm{Gr}$ as working electrode, saturated calomel electrode (SCE) as reference electrode, and platinum wire as counter electrode. The electrochemical characterization of the bare and modified Gr electrodes was performed in a PBS buffer at $\mathrm{pH} 7.0$, with $5 \mathrm{mM}\left[\mathrm{Fe}(\mathrm{CN})_{6}\right]^{-3 /-4}$ as electrochemical probe. The charge transfer process at the electrode/electrolyte interface of the modified electrode was studied by EIS technique, in the frequency range of $100 \mathrm{kHz}$ to $0.1 \mathrm{~Hz}$.

\section{Sample preparation}

The commercially available green tea sample of Lipton brand was purchased and used for experiments. The tea samples were first crushed to powder using a mortar, and then $0.1 \mathrm{~g}$ of dry powder was mixed to $100 \mathrm{~mL}$ of a $20 \%(\mathrm{v} / \mathrm{v})$ methanol solution. The mixture was shaken for $20 \mathrm{~min}$ at $80^{\circ} \mathrm{C}$, and filtered. The filtered solution was diluted to $100 \mathrm{~mL}$, and stored for further studies [24]. The catechol content in the tea sample extract was also quantified using standard HPLC method. The catechol content in $0.1 \mathrm{~g}$ of green tea powder extract was $0.279 \mathrm{mg} / \mathrm{g}$.

\section{Results and discussion AgNPs biosynthesis}

For the AgNPs synthesis, an aqueous solution of $\mathrm{AgNO}_{3}$ was mixed with the leaf extract of Convolvulus p., and incubated at room temperature. The reaction mixture initially showed a pale yellow color that gradually turned to dark brown, as the reaction proceeded, which suggested AgNPs formation through the reduction of $\mathrm{Ag}^{+}$ions to $\mathrm{Ag}^{0}$.

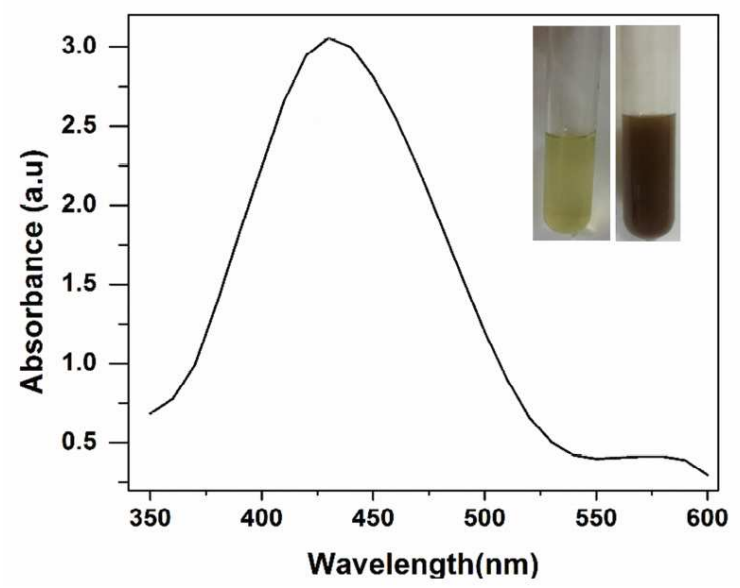

Figure 1. UV-vis spectra of biosynthesized AgNPs.

UV-visible spectroscopy was used as a tool to confirm AgNPs formation. The UV-visible spectral measurements of the reaction mixture at regular intervals 
during the incubation period showed a gradual increase in absorbance. The absorbance reached a maximum, and almost stabilized after 10 hours of incubation, indicating the completion of the reduction of silver ions to AgNPs. Further, a strong and broad peak in the UV-visible spectrum, around $420 \mathrm{~nm}$, clearly confirmed AgNPs formation (Fig. 1). This peak may be attributed to the surface plasmon resonance in AgNPs [25]. The UV-visible study clearly confirmed the completion of $\mathrm{Ag}^{+}$reduction and AgNPs formation in nearly 10 hours.

\section{PPO biosensor surface characterization using SEM}

The PPO biosensor was fabricated through the Gr electrode surface modification in successive steps by PPy electropolymerization, followed by the drop-casting of the AgNPs and PPO enzyme. At each modification step, SEM was used as an effective tool to analyze the surface morphology of the modified electrodes.
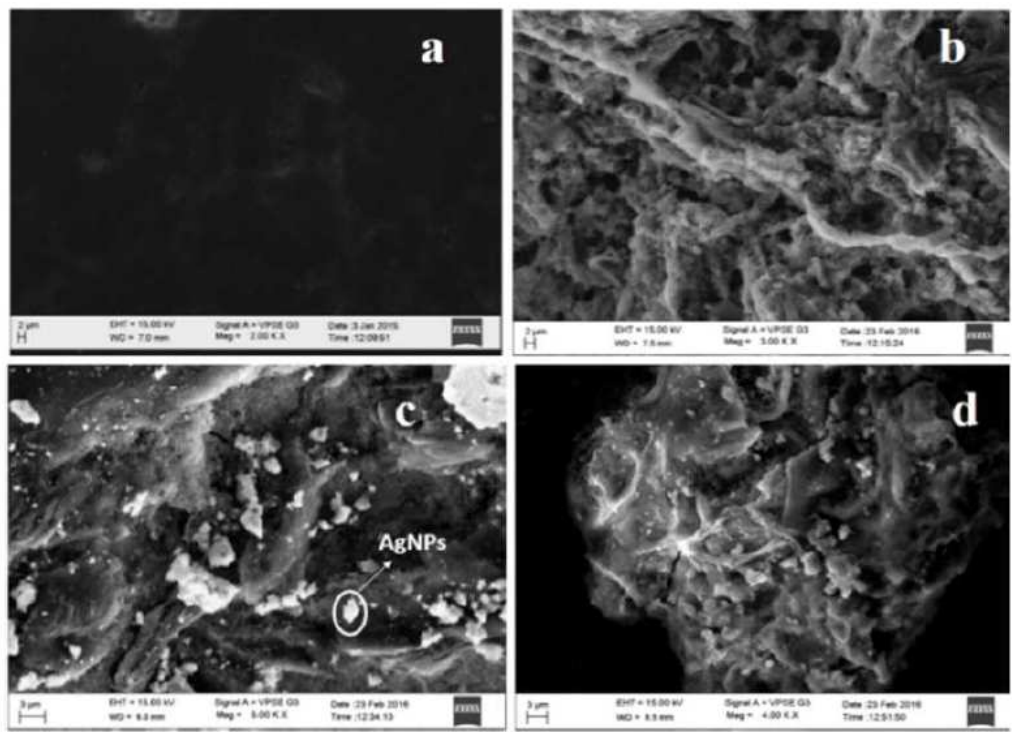

Figure 2. SEM images of a) Bare Gr, b) Gr/PPy, c) Gr/PPy/AgNPs and d) $\mathrm{Gr} / \mathrm{PPy} / \mathrm{AgNPs} / \mathrm{PPO}$ electrodes surface.

Fig. 2 shows the SEM images of (a) bare graphite, (b) modified Gr/PPy, (c) Gr/PPy/AgNPs and (d) Gr/PPy/AgNPs/PPO electrodes.

\section{PPO biosensor electrochemical characterization using CV and EIS}

The redox behavior of the bare and modified Gr electrodes was studied using 5 $\mathrm{mM}\left[\mathrm{Fe}(\mathrm{CN})_{6}\right]^{-3 /-4}$ as an electrochemical probe in PBS ( $\left.\mathrm{pH} 7.0\right)$, and compared after each modification step. From Fig. 3 A (a), it can be seen that the Gr electrode exhibits a pair of well-defined quasi reversible redox peaks, with a peak separation $(\triangle E \mathrm{E})$ of $133 \mathrm{mV}$. Comparatively, the CV of Gr/PPy [Fig 3A (b)] showed an increase in both oxidation and reduction currents, with a $\Delta \mathrm{Ep}$ of 125 $\mathrm{mV}$, because of the polymer presence, which exhibits a conducting property. Further, when the electrode was modified with AgNPs (Gr/PPy/ AgNPs), it showed an enhanced oxidation and reduction current with a $\Delta \mathrm{Ep}$ value of 108 $\mathrm{mV}$ [Fig 3.A (c)]. The enhanced conductivity was attributable to the highly 
conductive nature of AgNPs, which increased the electrode's surface area, and also facilitated the electron exchange between the electrode and the electrochemical probe. Finally, after the enzyme immobilization, the current at the $\mathrm{Gr} / \mathrm{PPy} / \mathrm{AgNPs} / \mathrm{PPO}$ electrode decreased with a $\triangle \mathrm{Ep}$ of $121 \mathrm{mV}$, indicating the enzyme's insulating property, which acted as a weak barrier to $\left[\mathrm{Fe}(\mathrm{CN})_{6}\right]^{-3 /-4}$ ion penetration [Fig 3.A (d)]. This confirmed the successful enzyme immobilization.
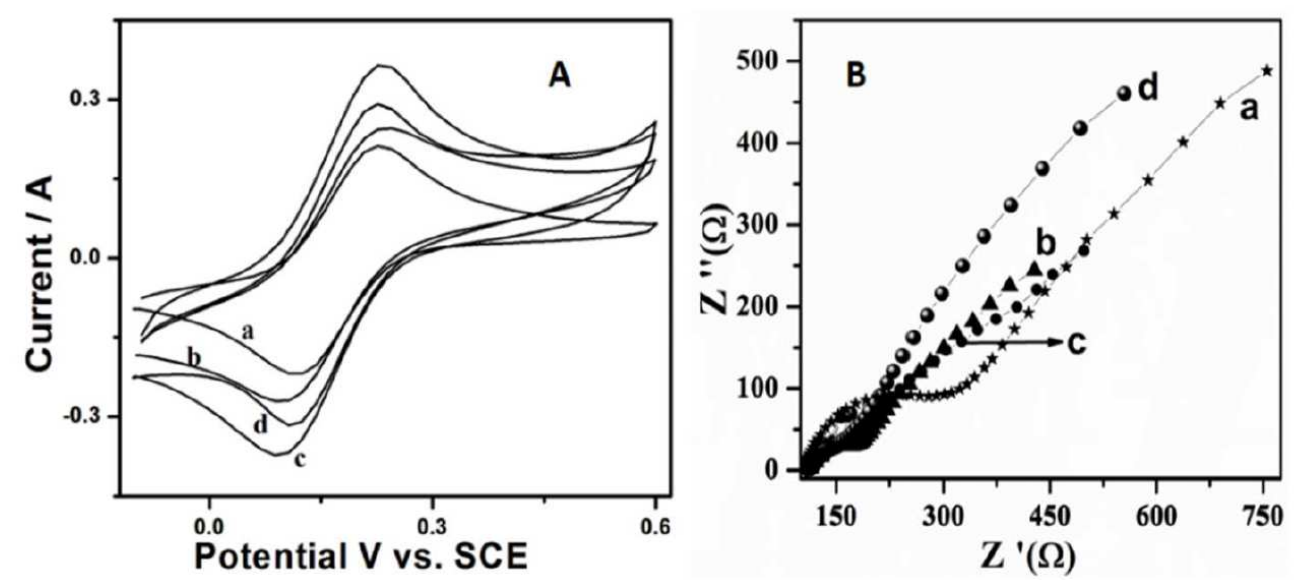

Figure 3. (A) $\mathrm{CVs}$ of a $5 \mathrm{mM} \mathrm{Fe}(\mathrm{CN})_{6}^{-3 /-4}$ solution obtained for a) bare $\left.\mathrm{Gr}, \mathrm{b}\right) \mathrm{Gr} / \mathrm{PPy}$, c) $\mathrm{Gr} / \mathrm{PPy} / \mathrm{AgNPs}$ and d) Gr/PPy/AgNPs/PPO. (B) Impedance spectra of a) bare Gr, b) $\mathrm{Gr} / \mathrm{PPy}, \mathrm{c}) \mathrm{Gr} / \mathrm{PPy} / \mathrm{AgNPs}$ and d) Gr/PPy/AgNPs/PPO.

EIS experiments were carried out to investigate the electrode surface features during each step of its modification. The charge transfer process occurring at the electrode/electrolyte interface was studied by carrying out EIS measurements in a frequency range of $100 \mathrm{KHz}$ to $0.1 \mathrm{~Hz}$, with an amplitude of $5 \mathrm{mV}$. Fig. $3 \mathrm{~B}$ shows the Nyquist plot of experimental data from EIS studies. The results of EIS studies indicate that the charge transfer resistance $\left(R_{c t}\right)$ of the Gr electrode was much higher $(200 \Omega)$ than that of the Gr/PPy electrode. The reduced Gr/PPy (170 $\Omega) R_{c t}$ value may be attributed to the electrode's increased surface area. The modified Gr/PPy/AgNPs showed a further decrease in $R_{c t}(47.89 \Omega)$ value, indicating the enhanced surface area and conductivity, due to the AgNPs incorporation to the electrode surface. Further, when the electrode was modified with the PPO enzyme, this resulted in an increased $R_{c t}(103.2 \Omega)$, which was due to the insulating property of the PPO enzyme. These results are in clear agreement with the CV results above discussed.

\section{Electrochemical detection of catechol using a developed Gr/PPy/AgNPs/PPO sensor}

The electrocatalytic properties of the developed PPO biosensor were examined by measuring its cyclic voltammetric response for different catechol concentrations $(0.001-0.009 \mathrm{mM})$, in an oxygen saturated PBS solution. 

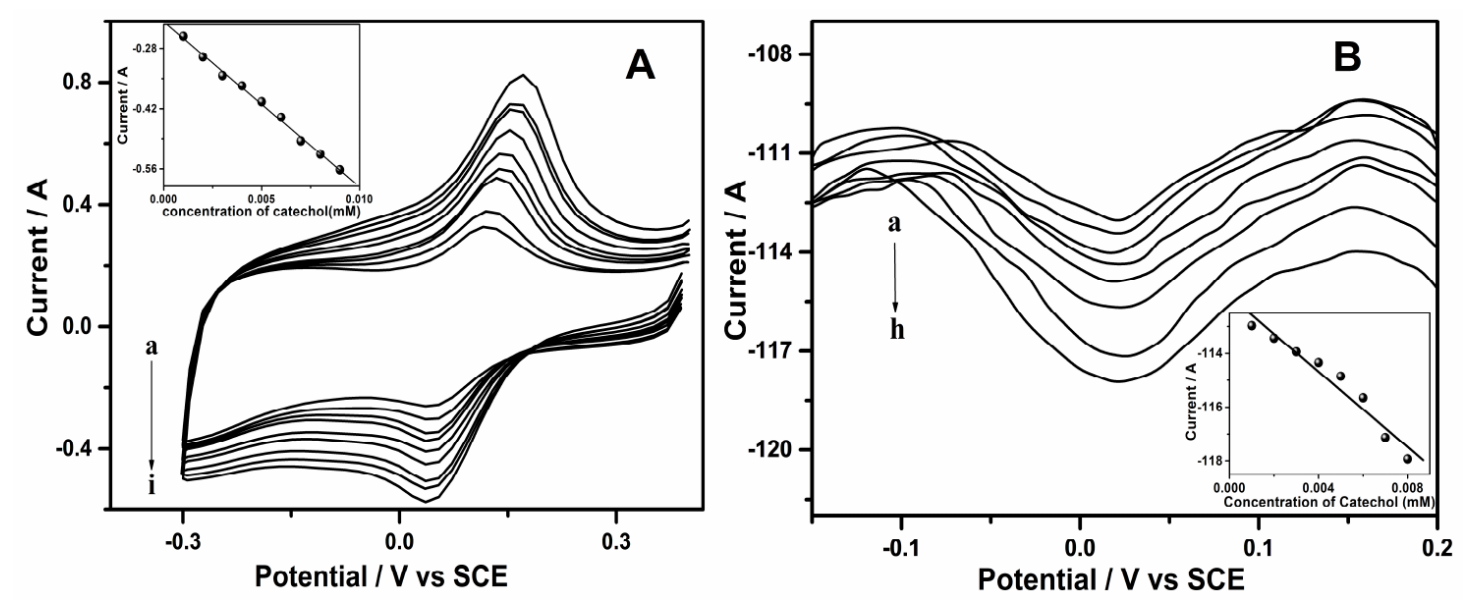

Figure 4. (A) Cyclic voltammograms of the Gr/PPy/AgNPs/PPO electrode with the successive addition of 0.001-0.009 mM catechol in 0.1 M PBS at $\mathrm{pH}$ 7. The inset shows the calibration plot of current vs. catechol concentration. (B) DPVs of the $\mathrm{Gr} / \mathrm{PPy} / \mathrm{AgNPs} / \mathrm{PPO}$ electrode at varying catechol concentrations $(0.001-0.008 \mathrm{mM})$ in a $0.1 \mathrm{M}$ PBS solution. The inset plot shows the calibration plot of the peak current versus catechol concentration.

The CV results in Fig. 4(A) show an increase in the reduction peak, with an increase in catechol addition to the oxygen saturated buffer solution.

The PPO catalytic reaction is as follows:

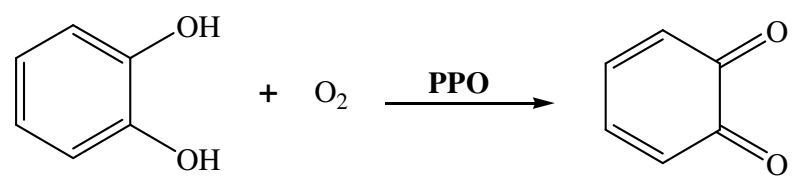

O-quinone formation was detected by amperometric current measurements, during the reduction at the electrode:

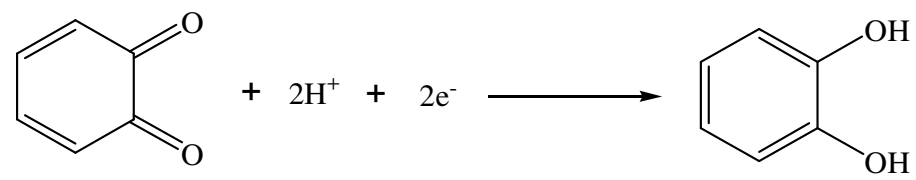

The cathodic peak current for the reduction process linearly increased with an increase in catechol concentration, in the linear range of 0.001 to $0.009 \mathrm{mM}$ (inset of Fig. 4 A), with a linear regression equation:

$$
I_{p c}(A)=-0.218-(-38.25) C_{\text {catechol }}(m M) ; R=-0.998
$$

DPV is a pulse technique that shows higher sensitivity, while detecting the lower analyte concentration. DPV experiments were performed for the $\mathrm{Gr} / \mathrm{PPy} / \mathrm{AgNPs} / \mathrm{PPO}$ electrode, in the potential range of +0.25 to $-0.2 \mathrm{~V}$. Fig. 4(B) shows a stable and well defined reduction peak, with an increase in catechol concentration. The calibration plot of $\mathrm{I}_{\mathrm{pc}} \mathrm{vs}$. catechol concentration was plotted, and the peak current was in linear relationship with catechol concentration in the range of 0.001 to $0.008 \mathrm{mM}$ (inset of Fig. 4 (B)), with a linear regression equation: 


$$
I_{p c}(A)=-111.8-(-700.7) C_{\text {catechol }}(m M) ; R=-0.974
$$

\section{Scan rate effect}

The effect of the scan rate on the cathodic peak current was studied, to investigate the nature of the electron transfer reaction occurring at the electrode surface. The study was carried out using $0.004 \mathrm{mM}$ catechol in $0.1 \mathrm{M} \mathrm{PBS}(\mathrm{pH}$ 7.0), for the different scan rates ranging from 10 to $100 \mathrm{mVs}^{-1}$, and the obtained results are shown in Fig. 5 (A). The results indicate that there was an increase in the oxidation and reduction peak current, with an increase in the scan rate from 10 to $100 \mathrm{mVs}^{-1}$. The linear regression equation was found to be:

$$
I_{p c}(A)=-0.113-\left(-1.004 \times 10^{-2}\right) v\left(V s^{-1}\right) ; R=-0.993
$$

The inset plot of Fig. 5(b) also indicates that the cathodic peak current proportionally varies to the square root of the scan rate with a linear regression:

$$
I_{p c}(A)=-0.303-(-0.136) v^{1 / 2} ;\left(V s^{-1}\right) ; R=-0.999
$$

These results suggest that the electrochemical reaction occurring at the $\mathrm{Gr} / \mathrm{PPy} / \mathrm{AgNPs} / \mathrm{PPO}$ electrode surface is an adsorption controlled reaction.

\section{pH effect}

The $\mathrm{pH}$ is one of the major parameters that influence the biosensor amperometric response. The $\mathrm{pH}$ effect on the $\mathrm{Gr} / \mathrm{PPy} / \mathrm{AgNPs} / \mathrm{PPO}$ biosensor performance was examined by measuring the current response of $0.004 \mathrm{mM}$ catechol in the $\mathrm{pH}$ range of 2 to 10. The amperometric response of the developed biosensor at different $\mathrm{pH}$ values is shown in Fig 5(B). The biosensor showed maximum response current at $\mathrm{pH} 7.0$, and the response decreased at high $\mathrm{pH}$ values, which could be attributed to the enzyme deactivation or leaching from the electrode surface [26].

\section{Temperature effect}

The temperature effect on the Gr/PPy/AgNPs/PPO modified electrode was examined over the temperature range of $10{ }^{\circ} \mathrm{C}$ to $70{ }^{\circ} \mathrm{C}$, in $0.004 \mathrm{mM}$ catechol. When the temperature was increased, the response current increased up to $40^{\circ} \mathrm{C}$, and then it gradually decreased with an increased temperature, as shown in Fig. 5 (C). This may be due to the enzyme deactivation beyond optimum temperature. However, for practical reasons, all of our experiments were performed at room temperature.

\section{Effect of interferences}

To test the practical applicability of the developed biosensor, a selectivity study was obligatory. Despite high enzyme specificity, the enzyme based sensors may give erroneous results for real samples, due to the interference from electroactive species present in such samples. Some common interferents, such as glucose, ascorbic acid and gallic acid, present in natural samples, may often interfere in 
catechol detection. The effect of these interferents in catechol detection was examined by measuring the reduction current on the subsequent addition of those interfering compounds. The sensor showed no significant changes due to the subsequent interferents' addition. This indicates that the developed $\mathrm{Gr} / \mathrm{PPy} / \mathrm{AgNPs} / \mathrm{PPO}$ biosensor is highly selective towards catechol detection.
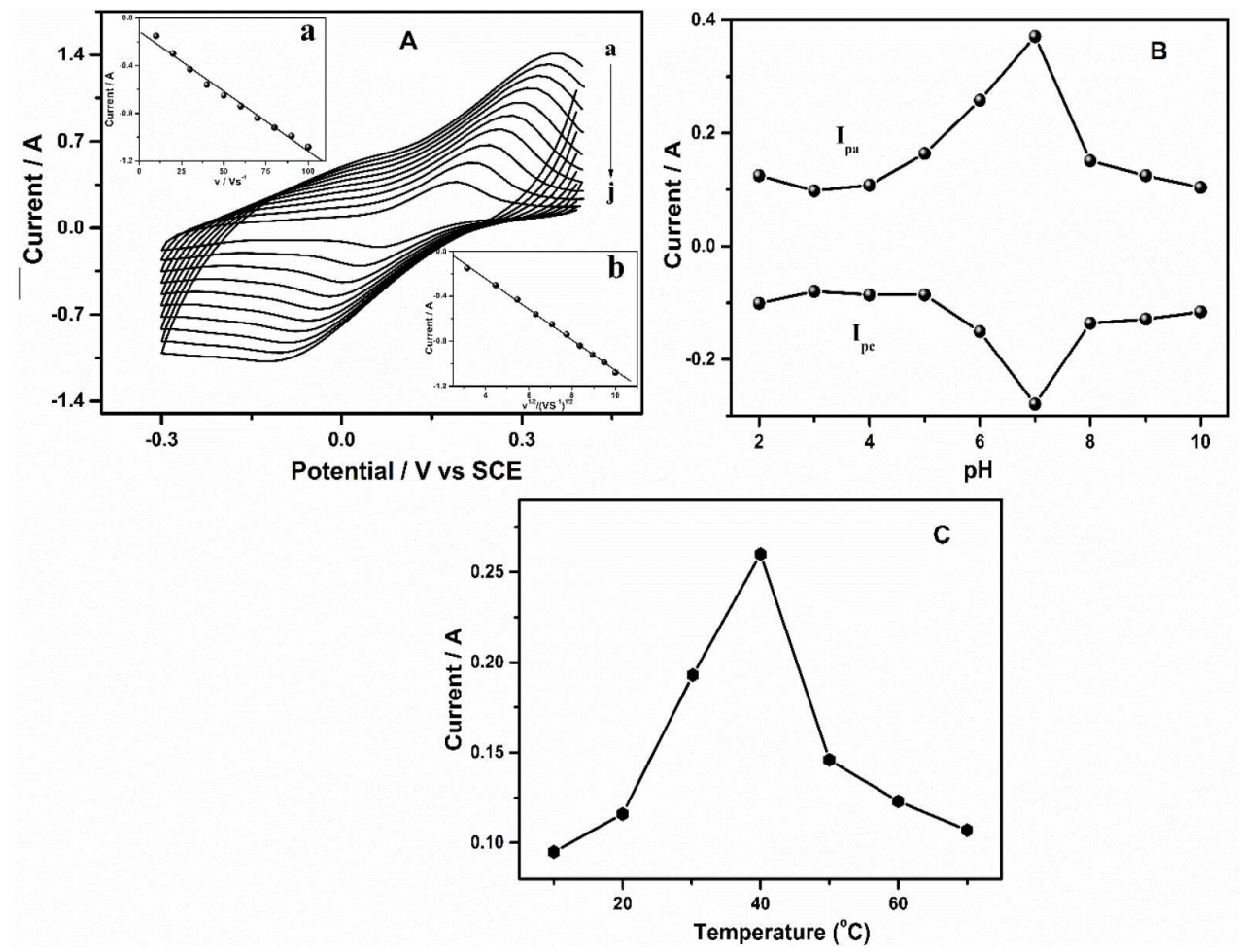

Figure 5. (A) Cyclic voltammograms of $\mathrm{Gr} / \mathrm{PPy} / \mathrm{AgNPs} / \mathrm{PPO}$ in $0.1 \mathrm{M} \mathrm{PBS}$ at $\mathrm{pH} 7$ containing $0.004 \mathrm{mM}$ catechol at different scan rates $\left(10-100 \mathrm{mVs}^{-1}\right)$; inset plots a) peak current vs. scan rate, b) peak current vs. square root of scan rate. (B) Effect of varying $\mathrm{pH}$ (from 2 to 10 ) on the peak current. (C) Effect of varying temperature (from 10 to $70{ }^{\circ} \mathrm{C}$ ) on the peak current.

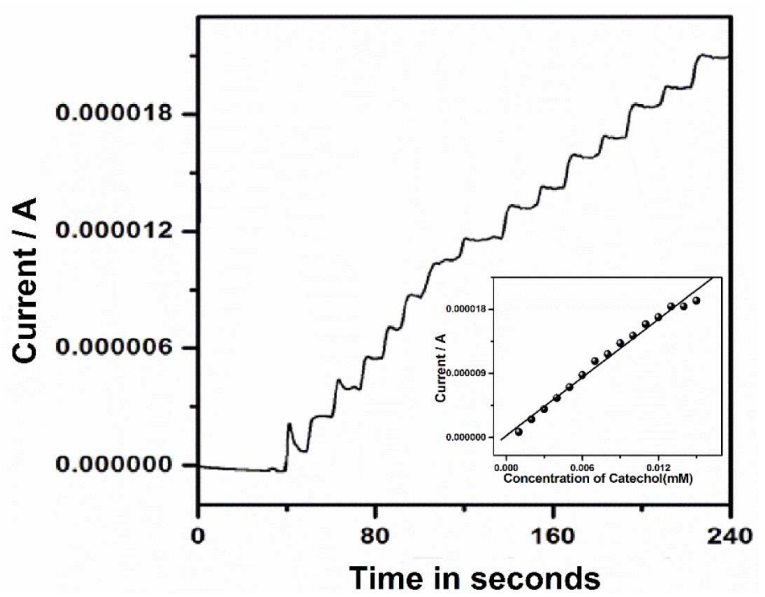

Figure 6. Chrono amperometric response of the Gr/PPy/AgNPs/PPO electrode in different catechol concentrations, at an applied potential of $+0.025 \mathrm{~V}$. The inset plot shows the calibration curve of the response current vs. catechol concentration. 


\section{Chrono amperometric response of the Gr/PPy/AgNPs/PPO biosensor}

Chrono amperometry is a widely used technique to evaluate the change in current response as a function of time. Fig. 6 shows the amperometric response of the enzyme modified electrode on the successive catechol addition in varying concentrations, at an applied potential of $+0.025 \mathrm{~V}$. The enhanced current was recorded with incremental catechol addition. The inset of Fig. 6 shows the current calibration plot as a function of catechol concentration. The chrono amperometric results indicate that the sensor exhibited a linear detection range of 0.001 to $0.015 \mathrm{mM}$, with a regression coefficient of 0.993 .

\section{Sensitivity and detection limit}

The detection limit of the developed sensor was determined according to:

$$
\frac{3 \sigma}{S}
$$

where $\sigma$ is the standard deviation of blank and $S$ is the slope of the calibration plot [27]. The detection limit of the sensor was found to be $0.47 \mu \mathrm{M}$, with a sensitivity of $13.66 \mu \mathrm{AmM}^{-1} \mathrm{~cm}^{-2}$, which is better, compared to that of previously reported works (Table 1). The sensor developed by Ameer Q. et al., using a PPy matrix, showed a detection limit of $1 \mu \mathrm{M}$, while the sensor in the present work, developed using AgNPs in the PPy matrix, showed a detection limit of $0.47 \mu \mathrm{M}$. On the basis of this study, it may be concluded that the sensor developed using a combination of biosynthesized AgNPs and PPO enzymes from Manilkara Zapota (sapota) is more effective in catechol detection

Table 1. Comparison of the proposed PPO biosensor with other PPO immobilized electrodes towards catechol detection.

\begin{tabular}{llcc}
\hline Electrode & $\begin{array}{c}\text { Linear range } \\
(\mathbf{m M})\end{array}$ & $\begin{array}{c}\text { Detection limit } \\
(\boldsymbol{\mu} \mathbf{M})\end{array}$ & Reference \\
\hline PPO entrapped in a garose-gaur gum & $0.06-0.8$ & 6.0 & {$[28]$} \\
IDE/PEDOT/CNT/PPO/GAD/HFR & $0.1-0.5$ & 4.2 & {$[29]$} \\
PPy/PPO & $0.001-0.016$ & 1.0 & {$[30]$} \\
Gr/PPy/AgNPs/PPO & $0.001-0.015$ & 0.47 & present work \\
\hline
\end{tabular}

\section{Repeatability and storage stability of the developed biosensor}

The stability and repeatability of the developed Gr/PPy/AgNPs/PPO sensor are the crucial factors for the sensor practical application. The repeatability of the developed sensor was investigated with a series of repetitive measurements using $0.004 \mathrm{mM}$ of catechol. The results of successive CV measurements showed that the sensor could retain $96 \%$ of its initial current response. This indicates that the developed biosensor has good repeatability. Furthermore, the shelf life/stability of the biosensor was also studied for a period of four weeks. The current response of the sensor decreased by, approximately, $2 \%$ of its initial value, in one week, and by $18 \%$, in four weeks. The decrease in response may be attributed to the partial enzyme denaturation. 


\section{Real sample analysis}

The developed sensor was examined for its effectiveness in real samples analysis, by using a green tea sample. The green tea sample was first analyzed using HPLC method, and it was found to contain $0.279 \mathrm{mg} / \mathrm{g}$ of catechol. The standard catechol and green tea sample HPLC chromatograms are shown in Fig 7. The tea sample extract was further diluted to get a $2 \mu \mathrm{M}$ catechol concentration, and later used for analysis using the developed sensor. The catechol amount detected by the $\mathrm{Gr} / \mathrm{PPy} / \mathrm{AgNPs} / \mathrm{PPO}$ sensor was $1.96 \mu \mathrm{M}$, against $2 \mu \mathrm{M}$ from the HPLC method, with an excellent recovery of $98 \%$. This indicates that the developed sensor can be effectively used for catechol detection in real samples. The developed sensor can be very useful in the tea industry for quality assessment of the tea samples, as catechol is one key component in deciding the tea's taste and aroma.
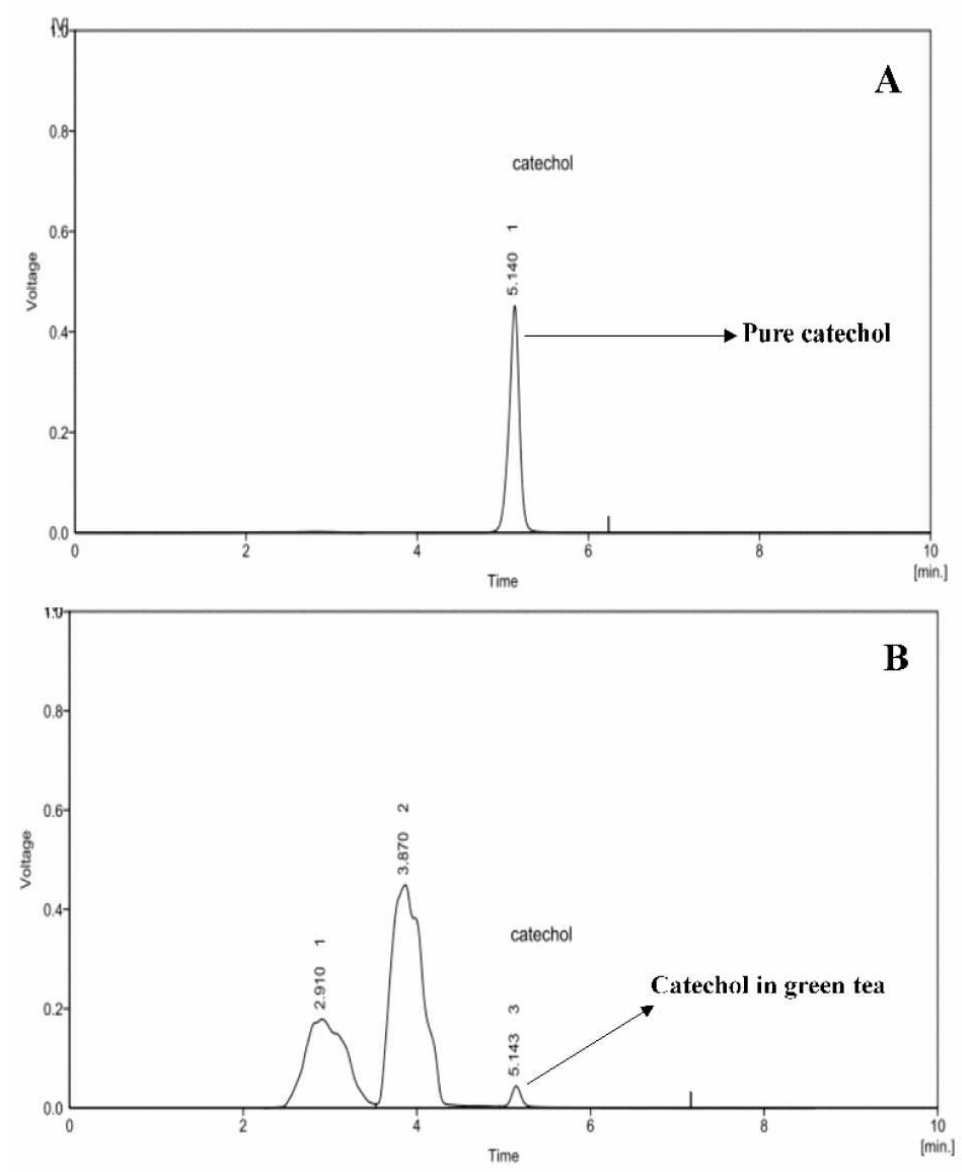

Figure 7. (A) HPLC chromatograms of standard catechol. (B) Green tea sample containing catechol. Catechol retention time: $5.14 \mathrm{~min}$.

\section{Conclusion}

The proposed Gr/PPy/AgNPs/PPO sensor, employing biosynthesized AgNPs from Convolvulus $p$. and PPO extracted from Manilkara Z. (sapota), exhibited a better detection limit than that of some of the sensors reported in literature. The real sample analysis showed an excellent recovery of $98 \%$, which indicates that 
the development sensor is efficient enough to analyze real samples. Furthermore, the sensor performance does not require any sample pretreatment. Therefore, on the basis of the above results, it is concluded that the proposed sensor can be effectively employed in catechol analysis in samples.

\section{Acknowledgement}

The authors gratefully acknowledge the financial support received from the Department of Atomic Energy-Board of Research in Nuclear Sciences, BARC, Government of India.

\section{References}

1. Li M, Ni F, Wang Y, et al. Electroanalysis. 2009; 21(13): 1521-1526.

2. Sun YG, Cui H, Li YH, et al. Talanta. 2000; 53(3): 661-666.

3. Wang G, He X, Zhou F, et al. Food Chem. 2012; 135(2): 446-451.

4. Lin H, Gan T, Wu K. Food Chem. 2009; 113(2): 701-704.

5. Subramanyam R, Mishra IM. Bioresour Technol. 2008; 99(18): 8917-8925.

6. Figueiredo EC, Tarley CRT, Kubota LT, et al. Microchem J. 2007; 85: 290296.

7. Moldoveanu SC, Kiser M. J Chromatogr A. 2007; 1141(1): 90-97.

8. Romani A, Minunni M, Mulinacci N, et al. J Agric Food Chem. 2000; 48(4): 1197-1203.

9. Fu J, Tan X, Shi Z, et al. Electroanalysis. 2016; 28: 203 - 210.

10. Apetrei IM, Rodriguez-Mendez ML, Apetrei C, et al. Sens Actuat B. 2013; 177: $138-144$.

11. Janegitz BC, Medeiros RA, Rocha-Filho RC, et al. Diamond Relat Mater. 2012; 25: 128-133.

12. Fiorentino D, Gallone A, Fiocco D, et al. Biosens Bioelectron. 2010; 25(9): 2033-2037.

13. Lima F, Lucca BG, Barbosa AM, et al. Enzyme Microb Technol. 2010; 47(4): 153-158.

14. Vicentini FC, Garcia LL, Figueiredo-Filho LC, et al. Enzyme Microb Technol. 2016; 84: 17-23.

15. Zhang M, Mullens C, Gorski W. Electroanalysis. 2005; 17(23): 2114.

16. Patil RS, Kokate MR, Kolekar SS. Spectrochim Acta Part A. 2012; 91: 234238.

17. Bao Q, Zhang D, Qi P. J Colloid Interf Sci. 2011; 360(2): 463-470.

18. Xue H, Shen Z. Talanta. 2002; 57(2): 289-295.

19. Zhang T, Tian B, Kong J, et al. Anal Chim Acta. 2003; 489(2): 199-206.

20. Dubey NK, Kumar R, Tripathi P. Curr Sci. 2004; 86(1): 37-41.

21. Agarwa P, Sharma B, Fatima A, et al. Asian Pac J Trop Biomed. 2014; 4(3): 245-252.

22. Sandeep S, Santhosh AS, Swamy NK, et al. Adv Mater Lett. 2016; 7(5): 383389.

23. Velusamy V, Arshak K, Yang CF, et al. Am J Anal Chem. 2011; 2(03): 392. 
24. Wang G, He X, Zhou F, et al. Food Chem. 2012; 135(2): 446-451.

25. Shahverdi AR, Fakhimi A, Shahverdi HR, et al. Nanomed Nanotech Biol Med. 2007; 3: 168.

26. Luo XL, Xu JJ, Du Y, et al. Anal Biochem. 2004; 334(2): 284-289.

27. Ntsendwana B, Mamba BB, Sampath S, et al. Int J Electrochem Sci. 2012; 7(4): 3501-3512.

28. Tembe S, Inamdar S, Haram S, et al. J Biotechnol. 2007; 128(1): 80-85.

29. Lete C, Lupu S, Lakard B, et al. J Electroanal Chem. 2015; 744: 53-61.

30. Ameer Q, Adeloju SB. Sens Actuat B. 2009; 140(1): 5-11. 